\title{
A new method for pore structure quantification and pore network extraction from SEM images
}

\author{
Chenhui Wang, ${ }^{*}{ }^{\dagger}$ Kejian Wu, ${ }^{\dagger}$ Gilbert G. Scott, ${ }^{\dagger}$ Alfred R. Akisanya, ${ }^{\dagger}$ Quan \\ Gan, ${ }^{\ddagger}$ and Yingfang Zhou ${ }^{\dagger}$
}

†School of Engineering, University of Aberdeen, Aberdeen AB24 3UE, United Kingdom $\ddagger$ Department of Petroleum Geology \& Geology, School of Geosciences, University of Aberdeen, United Kingdom

E-mail: r02cw17@abdn.ac.uk

\begin{abstract}
The structure of porous media is complex yet fundamental to the understanding of transport processes including single and multi-phase fluid flow. Pore network modelling is a valuable tool for studying these processes in many diverse porous media. This paper presents a new method of discretizing the pore space into connected pore elements which capture the pore geometry and topology. This method discards thedifference between pore bodies and throats, instead treats them equally as pore elements which captures the features of pore structure from the widest to the narrowest part of the pore space (pore body to pore throat), honours the topology and does not over-segment the pore space. The method is a higher level discretization of the pore space compared to other traditional methods which construct pore throats explicitly. Pore networks were extracted from three test images using the method and the computed flow properties are compared with finite element simulation in terms of absolute permeability. A reasonable agreement with finite element simulation is achieved, demonstrating the value and functionality of the new pore structure quantification method.
\end{abstract}




\section{Nomenclature}

\section{Acronyms}

FEM Finite element method

PNM Pore network model

PSD Pore size distribution

SEM Scanning electron microscopy

\section{Greek Symbols}

$\mu \quad$ Fluid viscosity, $\mathrm{Pa} \cdot \mathrm{s}$

$\sigma \quad$ Standard deviation of pore elements radius, $\mu \mathrm{m}$

$\Phi(\mathrm{r})$ Cumulative probability for a certain pore element radius $\mathrm{r}>\mathrm{r}_{\min }, \%$

\section{Latin Symbols}

A Cross-sectional area, $\mathrm{mD}$

D, dist Euclidean distance between the centre of two maximal balls, $m$

F Formation factor

g Hydraulic conductance for the pore elements, $\mathrm{m}^{4} /(\mathrm{Pa} \cdot \mathrm{s})$

G, $\hat{G}$ Shape factor

$\mathrm{g}_{i j} \quad$ Harmonic mean conductance between two connected pore elements, $\mathrm{m}^{4} /(\mathrm{Pa} \cdot \mathrm{s})$

$\mathrm{k} \quad$ Absolute permeability, $\mathrm{m}^{2}$

$\mathrm{k}_{\text {calc }}$ Calculated absolute permeability, mD

$\mathrm{k}_{\text {meas }}$ Measured absolute permeability, $\mathrm{mD}$ 
$\mathrm{L} \quad$ Length of pore element, $\mathrm{m}$

$\mathrm{N} \quad$ Coordination number

$\mathrm{p} \quad$ Perimeter, $\mathrm{m}$

P Fluid pressure, $\mathrm{Pa}$

$\mathrm{R} \quad$ Image resolution, $\mu \mathrm{m} /$ pixel

$\mathrm{R}^{2} \quad$ Correlation coefficient

$r_{0} \quad$ Cut-off pore element radius used in cumulative PSD curve fitting, $\mu \mathrm{m}$

$r_{c} \quad$ Characteristic pore element radius, $\mu \mathrm{m}$

$\mathrm{r}_{m b} \quad$ Radius of maximal ball, $\mathrm{m}$

$r_{\text {mean }}$ Geometric mean pore elements radius, $\mu \mathrm{m}$

$\mathrm{r}_{p \text { Curr }}$ Radius of the current pore element, $\mathrm{m}$

$\mathrm{r}_{p N e x t}$ Radius of the pore element to be found next, $\mathrm{m}$

a, b, c The fitting parameters for the cumulative PSD curve

\section{Subscripts}

fn Final nodes

$\mathrm{i}, \mathrm{j} \quad$ Pore element $\mathrm{i}$ and $\mathrm{j}$, or pore element coordinates min, max Minimum, maximum

n, b Nodes and bonds

pn Potential nodes 


\section{Introduction}

Over the past two decades, pore-scale modelling has developed rapidly as a predictive tool of multiphase flow which has been applied in petroleum engineering to deal with the assessment of conventional and unconventional hydrocarbon resources and in environmental engineering such as carbon dioxide capture and storage. ${ }^{1-11}$ With advances in computation and imaging technologies, pore scale models are increasingly being used in the oil industry to predict transport properties such as relative permeability and capillary pressure from micro scale rock images. ${ }^{12}$ Pore structures of reservoir rocks are complex and their understanding is fundamental to model fluid flow in porous media. Gaining a better understanding of single and multi-phase flow relies largely on quantifying the pore structures from 2D and $3 \mathrm{D}$ images, e.g. SEM and micro computed tomography (micro-CT).

Pore network modelling is an efficient way to simplify the computational procedures to simulate multiphase flow at the pore scale. ${ }^{13-17}$ A pore network model replaces the complex pore structures with a representative network of pore bodies connected by narrower restrictions called pore throats. Pore bodies and throats are assigned the corresponding geometrical properties, such as pore volume, length, inscribed radius, shape factor, etc. Thus the pore space can be segmented and the computational efficiency of fluid flow can be improved significantly.

Three main approaches ${ }^{8,10,18-23}$ have been proposed to extract the geometrical and topological properties of pore bodies and throats from 2D and $3 \mathrm{D}$ images. The first is the medial axis method which starts by constructing the medial axis or skeleton of the pore space. ${ }^{8,18}$ Junctions in the medial axis are then identified as nodes and the residual parts of the medial axis are identified as bonds. The second method is the maximal ball method, ${ }^{10,24}$ which represents the pore space by clusters of maximal balls, the largest spheres represent pores and the smaller connecting spheres define throats. The third method applies a watershed algorithm to the distance map of the pore space. ${ }^{22,23}$ The watershed basins define the pore bodies and the ridgelines or boundaries between basins define the pore throats. 
Basically, these three methods are able to capture the widest and narrowest parts of the pore space and thus multiphase flow simulation results can be in good agreement with experiments. ${ }^{25}$ A more detailed discussions on these three methods will be given in next subsection. However, pore network simulators treat pore bodies and throats slightly differently and most network extraction methods have a somewhat arbitrary distinction between pore bodies and throats. In the later section, a new method is proposed to address this issue

In the methodology section, the procedures to improve the pore structure quantification and pore network extraction are presented and the issues concerning pore bodies and throats are discussed. In the results section, a set of 2D SEM images are analysed to demonstrate the value and functionality of the pore structure quantification method. A finite element method was also used to validate the pore network constructed by our method. Finally, the conclusions and future work of this project are summarized.

\section{Medial axis method}

Themedial axis method was developed to characterize thetopology of pore space images. ${ }^{18,26}$ Jiang et al. ${ }^{8}$ developed a method to extract a pore network model based on the medial axis. They employed a thinning algorithm by scanning foreground pixels in ascending distance order and deleting as many pixels as possible to improve the thinning speed. The main advantage of the medial axis method is that it strictly preserves the topology of the pore structure. However, the distinction between pore bodies and throats is ambiguous. As shown in Figure 1(a), Jiang et al. ${ }^{8}$ regarded the pores as centering at junctions of the medial axis and the radius of a pore is the distance from the junction to the nearest boundary. However, this approach underestimates the size of pores and results in the identified pores not coinciding with the wider geometrical space of pores ${ }^{25}$ In addition, there is a problem if there is a second medial axis junction within the node radius. To address this, they introduced empirical node merging criteria, however, the overlapping problems of nodes still cannot be avoided. 


\section{Maximal ball method}

The concept of maximal balls was firstly proposed by Silin and Patzek ${ }^{24}$ to find the dimensionless capillary pressure in a drainage process rather than for extracting pore networks. The maximal balls are clustered into pore throat chains according to their size, rank and the other maximal balls they overlap with. This concept was extended by Al-Kharusi and Blunt ${ }^{27}$ to extract pore networks by introducing a more comprehensive set of criteria. Subsequently, Dong and Blunt ${ }^{10}$ developed a two-step searching algorithm and clustering processes to build pore network model. The main advantage of maximal ball method is that it can capture the wider pore space region corresponding to the geometrical pores and the concept is easy to understand.

However, there are still some minor problems related to the maximal ball method. ${ }^{10}$ This method tends to find too many small pores and throats which are down to the image resolution. ${ }^{1,25}$ In addition, this method is likely to underestimate the length of pores and throats when the pore throat chain is tortuous, see Figure 1(b). The reason is because the maximal ball method ${ }^{10}$ uses Euclidean distance between the centre of pores and throat to define the lengths. Again, the other problem is the distinction between pores and throats where some arbitrary criterion are used to partition the pore space. ${ }^{10}$

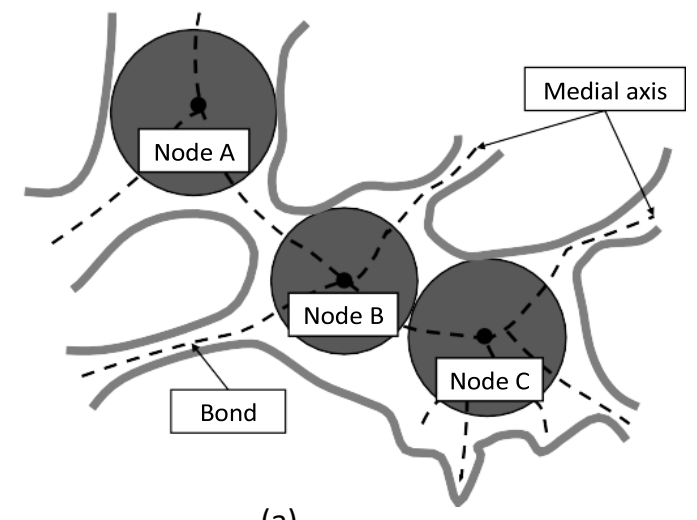

(a)

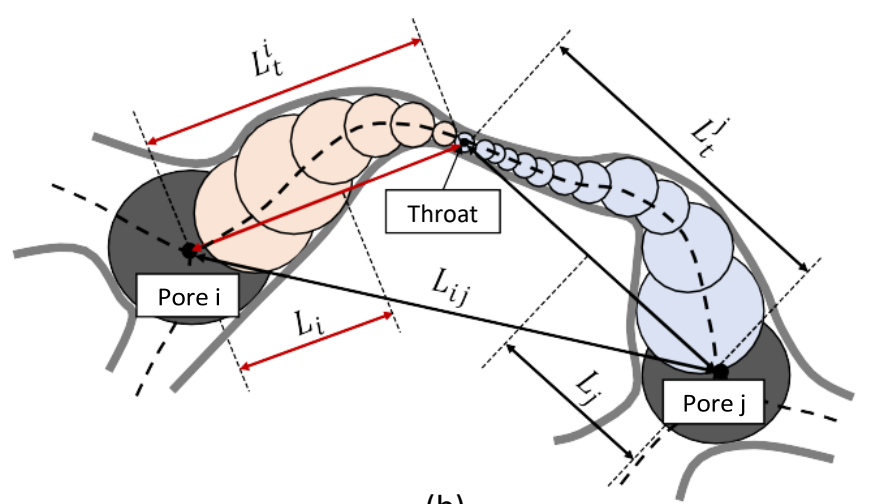

(b)

Figure 1: (a) Illustration of the medial axis method, ${ }^{8}$ the grey thick line is pore space boundary and the black dash line is the medial axis. (b) Schematic of the lengths of pores defined by maximal ball method. ${ }^{10}$ 


\section{Watershed segmentation method}

Watershed segmentation of porespacewasfirstly studied byThompson etal. ${ }^{21}$ and Sheppard et al. ${ }^{28}$ over ten years ago. Recently, this method has received more attention. ${ }^{22,23}$ Watershed segmentation utilizes the distance map of the pore space, which is defined as the distance from the centre of each pixel in pore space to the nearest pixel on the pore wall. Watershed segmentation transforms the pore space based on the distance map into catchment basins which comprises all points whose path of steepest descent terminates at thelocal minimum. The identified watersheds or surfaces of minimum distance represent the throats and the catchment basins are the pore bodies. All of the pore volume is assigned to pore bodies and throats are surfaces that define the boundary between two pores.

Recently, Gostick ${ }^{23}$ employed the marker-based watershed segmentation to extract pore networks. This method applies several improvements to eliminate the spurious or erroneous peaks (local maxima in the distance map) which cause the over-segmentation problem particularly in high-porosity materials. The main advantage of the watershed segmentation method is its efficiency in constructing pore networks. However, it is less reliable when dealing with sheet-like pores where the distance map is not a good representation of the pore space. ${ }^{29}$ In addition, watershed segmentation is sensitive to surface roughness which creates spurious local maxima. Although some spurious local maxima can be removed by filters, the method needs user-supervision and is hard to apply in automatic pore space segmentation.

\section{Methodology of pore space quantification}

In the current study, we propose a general method to quantify pore structures and construct pore network based on micro-CT or SEM images. The method, which involves discretizing the pore space into a set of connected pore elements to capture the pore geometry and topology, combines the strengths of maximal ball method ${ }^{10}$ and medial axis method ${ }^{8}$ but overcomes their problems. The resultant pore network of this method is applicable to sin- 
gle phase and multi phase transport properties, e.g., calculation of absolute permeability, capillary pressure curves and relative permeability.

Work-flow of pore structure quantification and pore network extraction method

Input: SEM or Micro-CT rock images

Output: Geometrical and topological properties of pore elements and pore element network

Step 1: Preliminary processes for the grey scale rock images, including thresholding, open and close processes and median filtering.

Step 2: Calculate the Euclidean distance map of void space, i.e., the distance of each pixel inside the pore space to the nearest boundary pixel of pore space and solids.

Step 3: Obtain the medial axis of the pore structure by the modified Zhang-Suen thinning algorithm, ${ }^{30,31}$ and make sure the generated medial axis has single pixel width and is centred inside the pore space.

Step 4: Apply the novel criteria to deal with the junctions of medial axis to define effective pores coinciding with wider geometrical pore bodies. The details of the procedures are shown in Algorithm 1.

Step 5: Topologically track the residual medial axis chains, determine the pore element positions and deal with the final pore elements on type-I medial axis chains to avoid overlap. Step 6: Partition the pore space based on common strings of the two connected pore elements.

Step 7: Compute the geometrical and topological properties of the partitioned pores, including inscribed radius, length, area, coordination number, etc.

Step 8: Employ the connected pore elements to construct a pore network.

\section{Preliminary processes}

The first step is to apply a threshold to convert the greyscale SEM image to a binary image. The threshold value is selected subjectively to retain most of the features of the pore structure. Figure 2(a) shows an example SEM image and Figure 2(b) is the segmented binary image where black regions are pore space and white regions are rock matrix. We did not investigate the sensitivity of the results to the threshold value because this issue has been addressed by several previous studies. ${ }^{32,33}$ The resulting image after thresholding still has significant noise, hence the opening and closing digital morphology processes are employed to remove the noise and fill small holes on the binary image. We use a mask size of 3 pixels for both opening and closing processes. In addition, a median filter with a size of 
3 pixels is employed to smooth the pore surface because the thinning algorithm used later is sensitive to the surface roughness. Weuse functions from $\mathrm{OpenCV}^{34}$ to conduct preliminary processes. Figure 2(c) shows the image after preliminary processes and the pore structures are maintained.
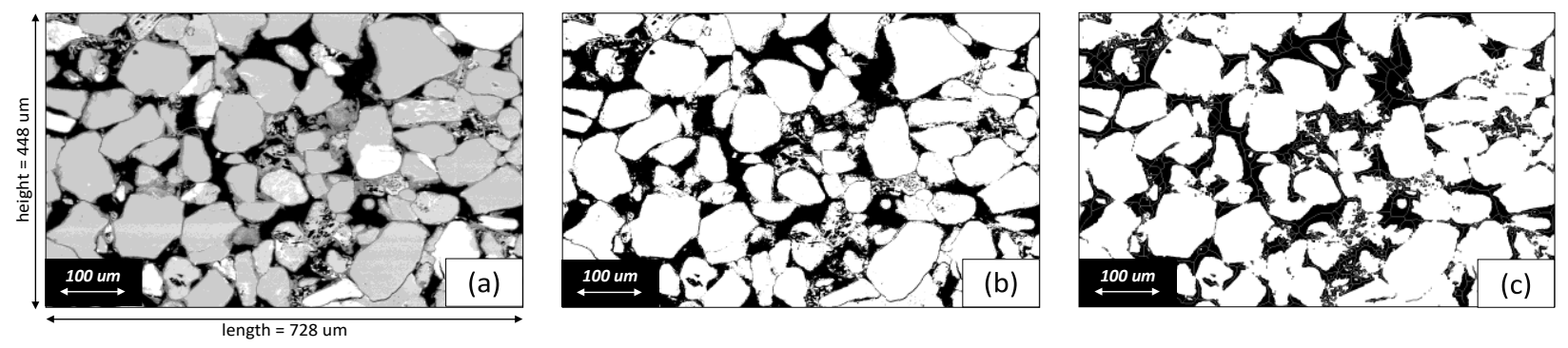

Figure 2: Core sample 210: (a) SEM image with resolution of $0.52 \mu \mathrm{m} /$ pixel was obtained from a North Sea sandstone reservoir. (b) Binary image after applying threshold of 140, whereblackzones are pores and white aregrains. (c) Binaryimage after applying preliminary processes mentioned above, and the corresponding medial axis generated by the modified Zhang-Suen thinning algorithm.

\section{Distance map calculation and medial axis transformation}

The distance map is the Euclidean distance from the centre of each pixel in pore space to the nearest pixel between boundary of pore space and solids. We use the function "distanceTransform" of OpenCV ${ }^{34}$ to compute distance map.

In this work, the Zhang-Suen thinning algorithm ${ }^{30}$ is employed to obtain the medial axis. The single pixel width of the medial axis is achieved by applying deleting templates summarized from the redundant pixels on the medial axis. ${ }^{31}$ The generated medial axis satisfies a single pixel width criterion which is necessary for the topological determination of pore elements locations.

\section{Pore structures quantification by pore element}

Weintroduce the pore element as the maximal inscribed circle in 2D, which can be clustered into nodes and bonds for the convenience of the pore network concept. However, it may not 
actually be necessary to make a distinction between nodes and bonds and we will discuss this issue later on. Firstly, we focus on the junctions of the medial axis and set several criteria on the one hand to capture the wider geometrical pores and on the other hand to avoid the overlap with other pore elements. Secondly, we topologically track the residual medial axis chains to determine the position of residual pore elements. The details of the algorithm involved in step 4 and 5 are shown in Algorithm 1 and 2 respectively.

We label all of the pixels on the medial axis with more than three neighbouring pixels (known as junctions of the medial axis) based on the 8-connected neighbour scheme for 2D image. These pixels are sorted by their radius and saved into the potential nodes vector. We search the $2 \times \mathrm{r}_{m b}$ radial range pore space for each element in the potential nodes vector. The objective is to ensure the identified nodes coincide with the wider geometric pore space and avoid the overlap of nodes. To achieve this objective, we need to satisfy three constraints. The first constraint is to avoid the overlap with the final node (fn) elements. This is achieved by searching the pixel $(i, j)$ whose maximal ball radius satisfy $r_{m b(i, j)}+r_{f n}<\operatorname{dist}$ $(i, j), \frac{1}{\text {, }(f n . x, f n . y)}$. The second criterion is to ensure the junction pixels are included in the node element to be found, which is necessary for the following steps and ensures the residual medial axis chain can be tracked without duplication. The third constraint is to identify the element that has the maximal radius inside the search range. By applying the above criteria, theidentified nodes coincide with the wider region of geometrical pore bodies without overlapping. Figure 3 shows the results of four identified nodes. Instead of the overlapped potential nodes, the final nodes identified by these criteria do not overlap and can capture the wider pore space.

In addition, we assign each node a unique index and record pixels beside each node on the medial axis to determine the topology of the pore structure, which is shown as the blue dots in Figure 3. The identification of nodes ensures all branch points or junctions of the medial axis are contained within nodes. Therefore, the residual medial axis can be tracked recursively without duplication to determine the pore elements position. 


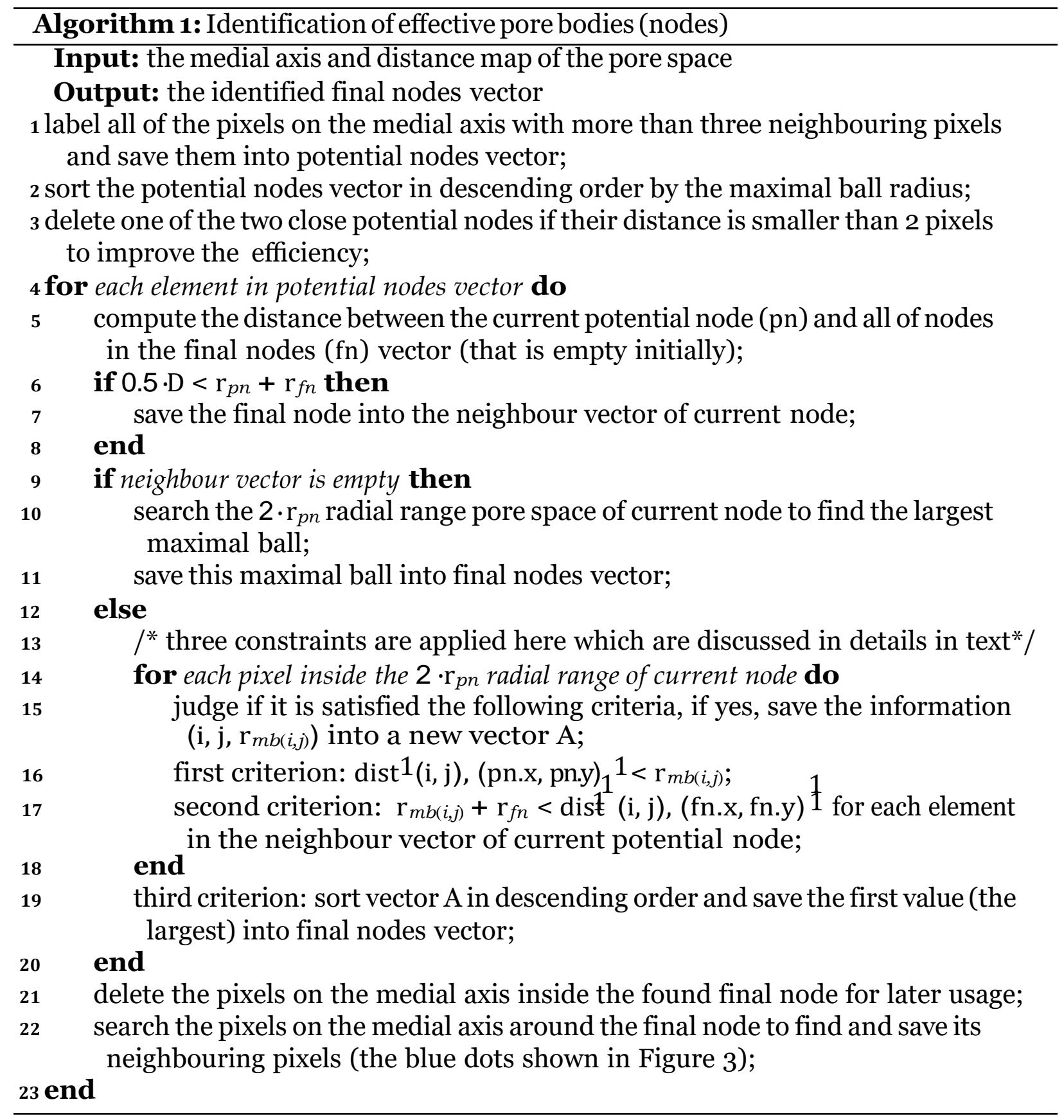




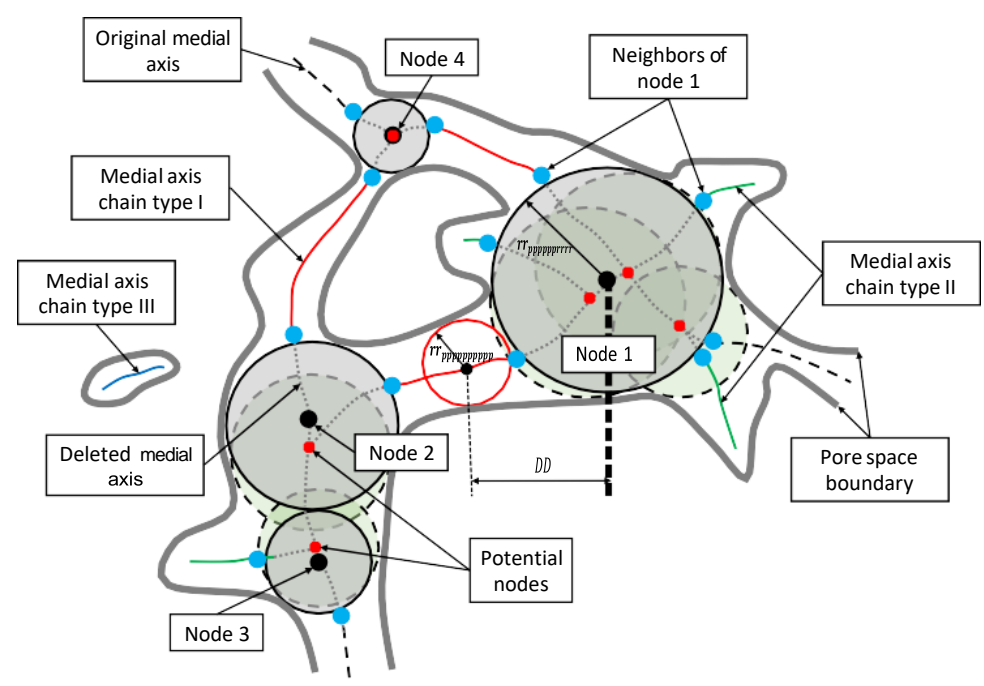

Figure 3: Illustration of nodes identified to capture the wider geometric pore space, procedures to track medial axis chains and determination of the pore elements positions, where green circles with dash dot outline and red dot centres represent the potential nodes, blue dots are the neighbouring pixels of the final nodes, and black dots are the centre of final nodes, which are exaggerated for vision.

Basically, there are three kinds of residual medial axis chains. Chain type I connects two nodes, chain type II connects one node and extends to dead end and chain type III connects zero node. To determine the pore elements position on these three kinds of medial axis chains, we start from the chain end that has larger radius for chain type I and III, but always start from the chain end that is close to the connected node for chain type II. Pore elements on the medial axis chains can be determined recursively, and the procedures are shown in Algorithm 2.

By examining each pixel on the medial axis chain, the pore element position is identified without overlap if it satisfies $D-r_{p C u r r}-r_{p N e x t} \subseteq[-1,1]$. The comparison range can be set wider, for example, [0,2] to avoid overlap between the two neighbouring pore elements. The red circle shown in Figure 3 is the identified pore element on the medial axis chain. We apply this procedure until all of the residual medial axis pixels are processed. Figure 4 shows an example of the pore elements identified to characterize the pore structure. Due to the nature of discretization and because the new method deals with the larger junction pores first and then fills the connecting throat pores, there are some pore elements which 


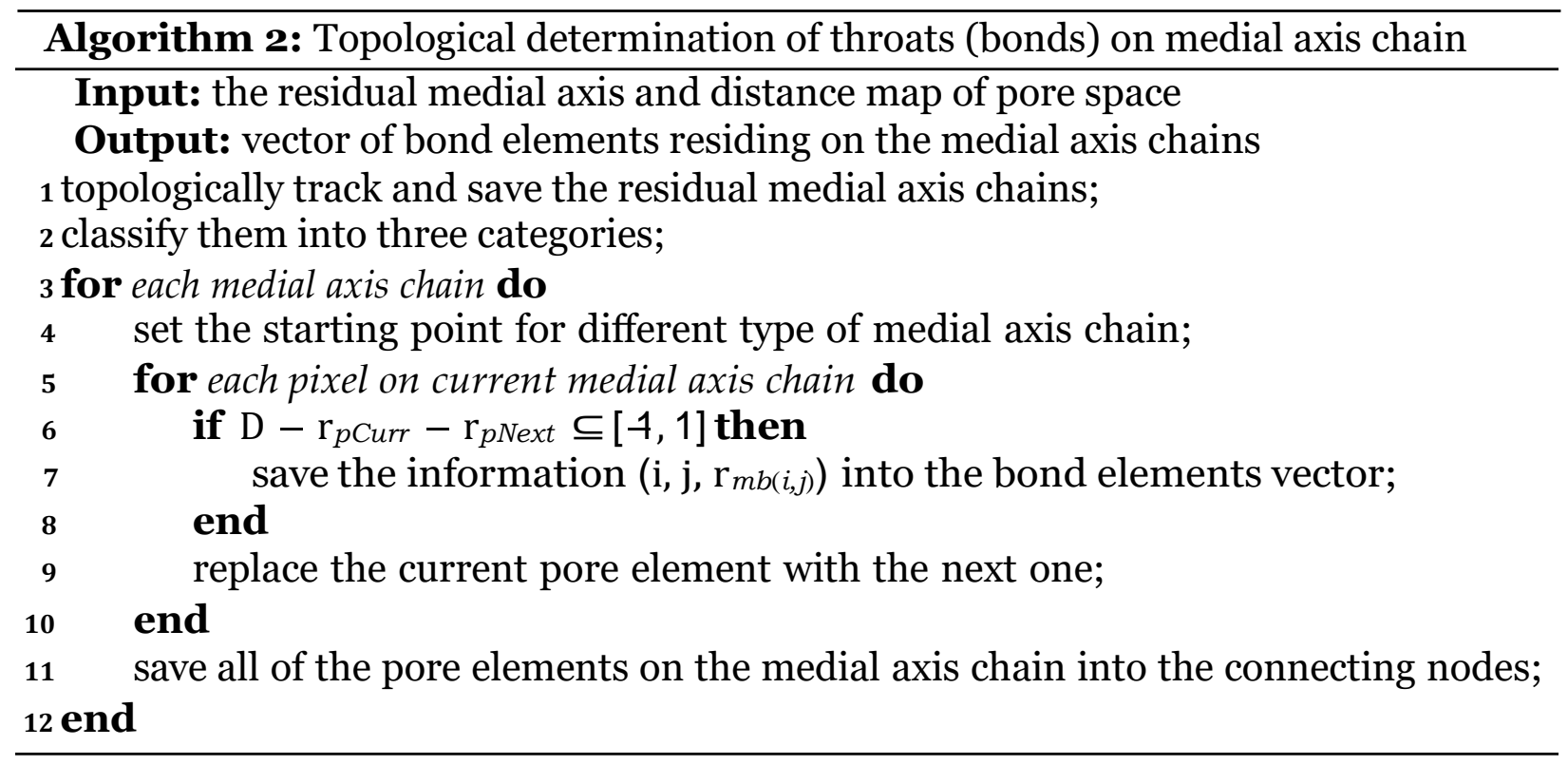

are not touching the pore space and solid boundary as shown in Figure 4 and some figures below. However, we record two radii for each pore element, one for plotting and the other for calculation. The former one, showing in the figures, is used to partition the pore space and compute the geometric properties of pore elements, while the latter one is the radius of maximal ball and is used to assign conductance and compute the permeability discussed later.

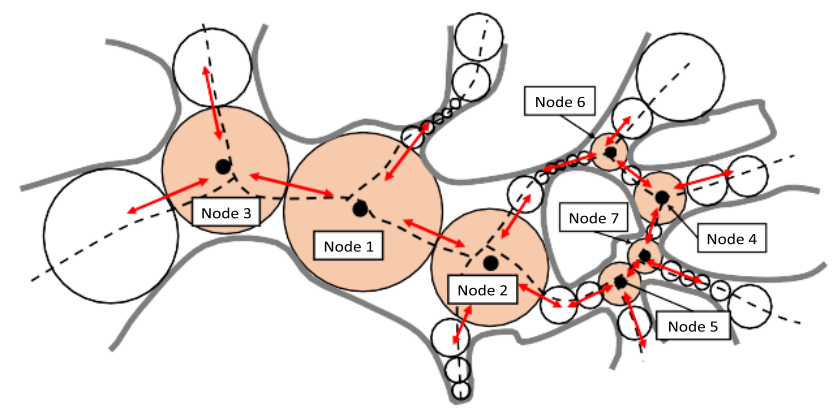

Figure 4: Illustration of the fitted pore elements by the new pore quantification method, where the circles represent the pore elements to characterize pore space, orange circles perform as junctions of fluid path, red arrows show the hierarchy of the subsets of pore element chains.

Theconnectivity of the porestructure is represented bythenodes connecting their neighbouring poreelements chains sets. All poreelements are quantified by thegeometrical prop- 
erties, including maximal ball radius, radius for plotting, area and the connectivity with neighbouring pore elements, etc. These properties are obtained by partitioning the pore space with the radical axis (or power line) between two neighbouring connected pore elements. The radical axis is a line perpendicular to the line connecting the centres of two non-concentric circles, which is obtained by subtracting the equations of the two circles. Algorithm 3 shows the procedures to partition the pore space. At the end of this process, the pore space is partitioned into a sequence of segments bounded by the radical axes. Figure 5 shows an example of the segmented pore space.

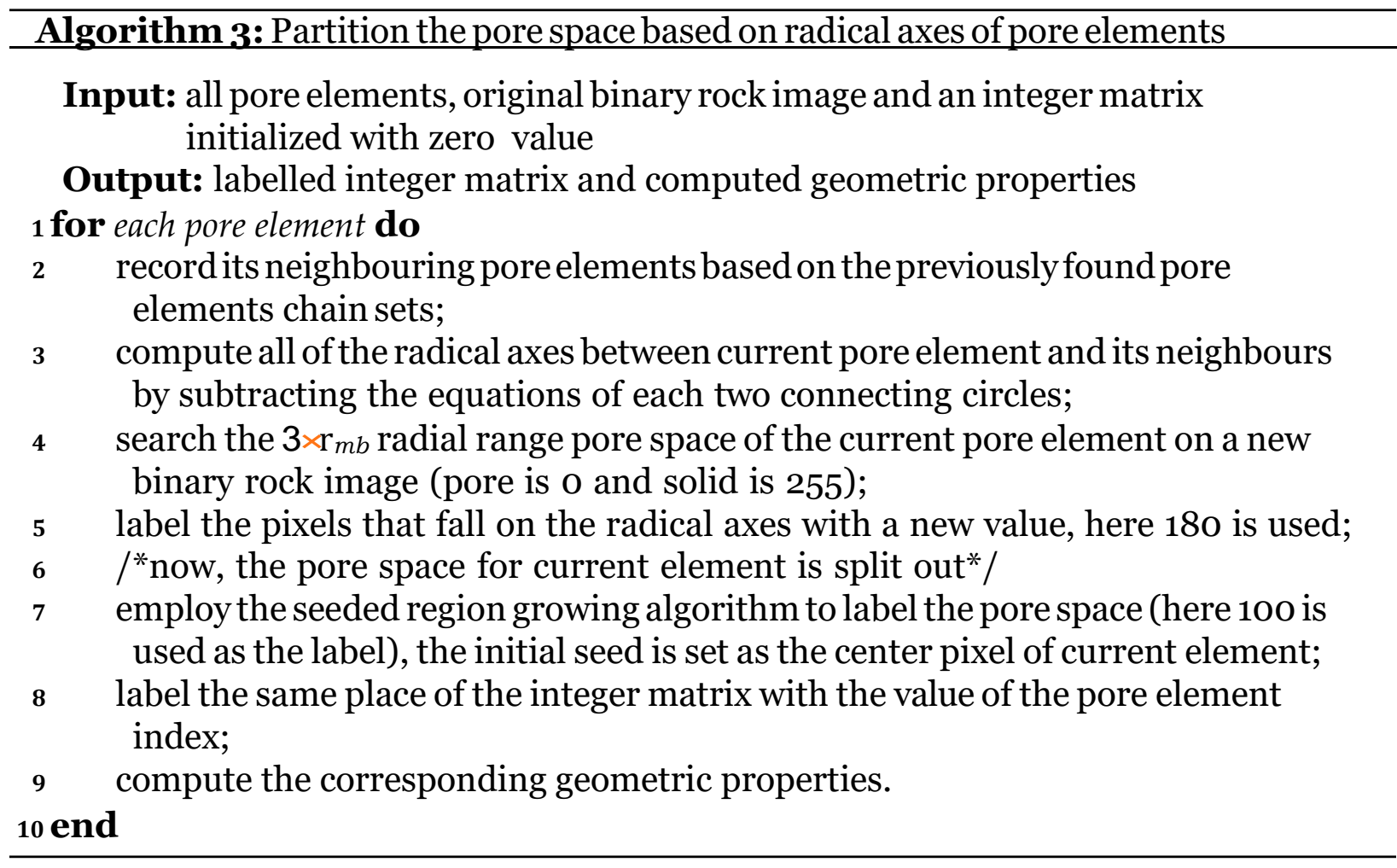

We note that our pore space partition method is different from that were used by Liang et al. ${ }^{35}$ and Jiang et al. ${ }^{8}$ Our method can avoid the problem of discretization error present in the method of Liang et al. ${ }^{35}$ who use the line perpendicular to the medial axis. In addition, our method can reduce the computational demands compared with the method of Jiang et al., ${ }^{8}$ since they checked 13 discrete directions for every pore element to determine the plane to partition the pore space. Moreover, the idea of radical axes can be easily extended 
to $3 \mathrm{D}$ case where the radical planes can be obtained by subtracting the equations of two neighbouring spheres.

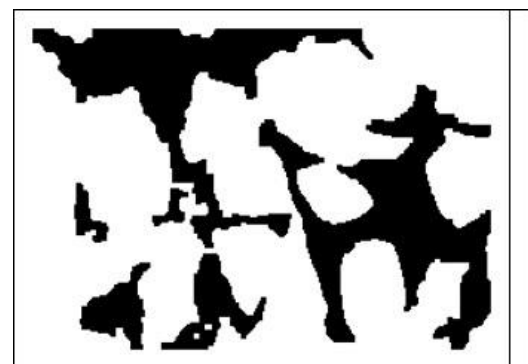

(a)

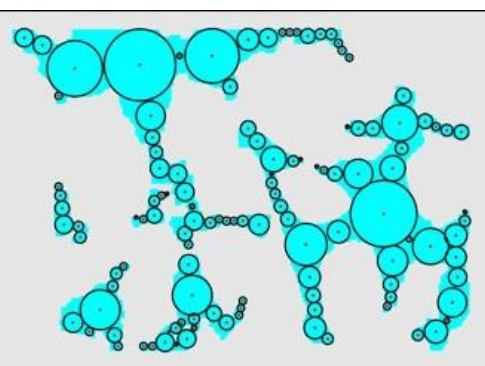

(b)

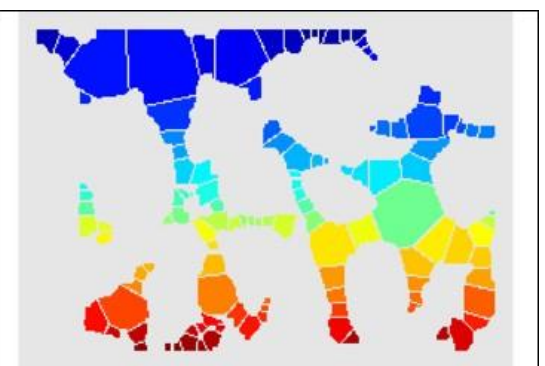

(c)

Figure 5: Example of pore space segmentation results. (a) Binary rock image, where black are pores and white are solids. (b) The pore elements found by the new method. (c) The segmented pore space based on radical axes method.

\section{Pore networks construction}

Having segmented the pore space into a set of pore elements, pore networks can be constructed to simulate fluid flow. The first issue is how to divide the pore space into pore bodies and throats. Although the pore network method has been successful in predicting transport properties, the question of how to set the boundary between pores and throats is still ambiguous. In the work of Dong and Blunt, ${ }^{10}$ they used a segmentation coefficient of 0.6 to partition the generated pore throat maximal ball chains while Øren and Bakke ${ }^{36}$ used a coefficient of 0.5 in their process based method.

Two critical questions to address in pore networks constructions are: (i) how does one chooses the segmentation coefficient and (ii) if it is important to differentiate the porebodies and throats. As Bultreys et al. ${ }^{25}$ pointed out, pore network models are more sensitive to pore and throat geometrical properties determination rather than how the pore space is initially split up. This indicates that what matters in the pore networkfluid flow simulation is whether the exact pore geometry features can be captured and assigned to pore elements rather than the differentiation between pores and throats. Therefore, we discard the difference between pore bodies and throats and treat them equally as pore elements. 
The pore elements determined by this new method are close-packed non-overlapping maximal circles. In fact, this method is a higher discretization of the pore space compared to the watershed method ${ }^{22}$ and the maximal ball method. ${ }^{10}$ For example, the watershed segmentation method partitions the pore space with the local minima, but our method continues partitioning the pore space within the pore bodies, which is a higher level discretization. We do not need to define the throats, because our method uses chains of pore elements to capture the changes of pore size, hence the wider and narrower geometric pore space features can be represented. This honours the topology and does not over-segment the pore space. Therefore, we use the pore elements directly to construct a pore network.

\section{Transport properties calculation}

Here, we show how to compute the absolute permeability from the pore element network. We consider the single phase incompressible flow of water in a porous medium. Constant pressure boundary condition is adopted for the inlet and outlet, and the other boundaries areassumed as no-flow boundaries. In addition, weassume the fluid flow is parallel to image plane rather than perpendicular to it as per a capillary tube bundle model. The latter only honours the pore size of porous media while our approach honours both the pore size and connectivity, which is known to be important. ${ }^{39}$ The Stokes equation (1) and mass balance equation (2) are solved.

$$
\begin{gathered}
\mu \nabla^{2} \overrightarrow{\mathrm{u}}-\nabla \mathrm{P}=0 \\
\rho \nabla \mathrm{u}=0
\end{gathered}
$$

There is a slight difference between the calculation of our pore element network and the traditional pore network, because we use the pore elements and discard the difference between pore bodies and throats. In our model, each pore element is a single calculation unit. As shown in Figure 6 (d), fluid pressures are defined at the centre of the pore elements and pressure drop occurs between the connected pore elements. The flow rate $Q_{i j}$ and the 
harmonic mean conductance $\mathrm{g}_{i j}$ between two connected pores elements $\mathrm{i}$ and $\mathrm{j}$ are given by:

$$
\begin{gathered}
\mathrm{Q}_{i j}=\frac{\mathrm{g}_{i j}}{\mathrm{~L}_{i j}}\left(\mathrm{P}_{i}-\mathrm{P}_{j}\right) \\
\mathrm{g}_{i j}=\frac{\mathrm{L}_{i j}}{\frac{L_{i}}{L_{i}}+\frac{L_{j}}{g_{j}}}
\end{gathered}
$$

Mass conservation is imposed for each pore element $\mathrm{i}$ :

$$
{ }_{j}^{N_{i}^{i}} \mathrm{Q}_{i j}=0
$$

wherej runs over all the poreelements connecting to pore element i. Combining the equation (3) and (5), a linear set of equations can be defined and solved for the pore pressures. The number of equations and the number of unknowns is equal to the number of elements. The system of equations is solved using the algebraic solver in OpenCV. ${ }^{34}$

With the pressure known at the centre of each pore elements, the total flow rate $Q_{t}$ is found by calculating the average flow rate along the inlet and outlet, and the absolute permeability $\mathrm{k}$ of the network is found from Darcy's law:

$$
\mathrm{k}=\frac{\mathrm{Q}_{t} \underline{\mu L}}{\mathrm{~A} \Delta \mathrm{P}}
$$

Assigning the hydraulic conductance for each pore element of the pore network in 2D images is different from that in $3 \mathrm{D}$ images. For the $2 \mathrm{D}$ case, the fluid velocity along the direction perpendicularto 2Dimage(zcoordinate) is a constant value. Therefore, weassume each pore element is apseudo-3D-rectangular-duct with alarger width (z coordinate). While for the $3 \mathrm{D}$ case, it depends on the real cross-sectional area and shape of each pore element 
perpendicular to the flow direction. 


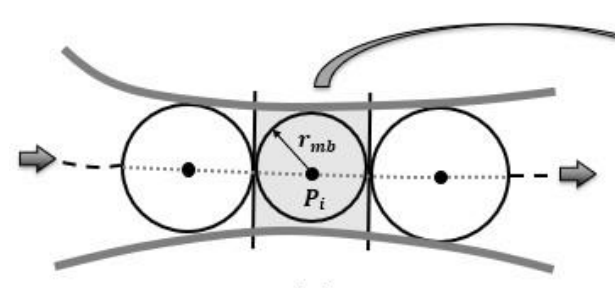

(a)

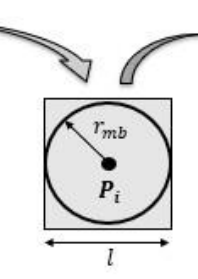

(b)

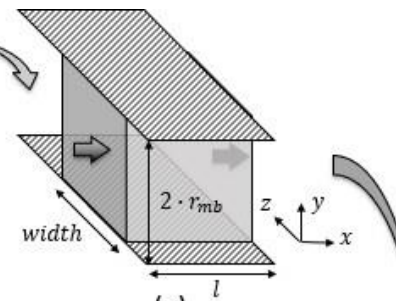

(c)

$$
\begin{array}{|l|}
\sum_{j}^{2} \frac{g_{i j}}{L_{i j}}\left(P_{j}-P_{i}\right)=0 \\
g_{i j(j=1)}=\frac{L_{i}+L_{i-1}}{\frac{L_{i}}{g_{i}}+\frac{L_{i-1}}{g_{i-1}}} \\
\hline
\end{array}
$$
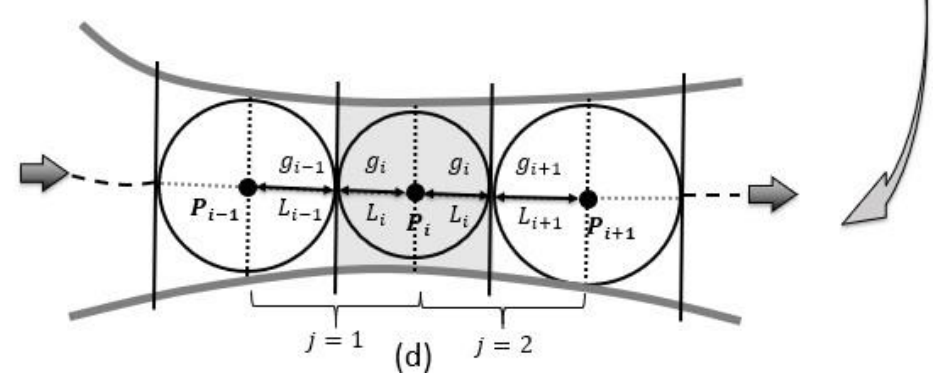

Figure 6: Illustrations of transformation of pore element to pseudo-3D-rectangular-duct and assignment of conductivity factor, where the arrows show the flow direction. (a) Three pore elements. (b) The partitioned pore space of the middle pore element, (c) The corresponding pseudo-3D-rectangular-duct of the middle pore element which is assumed as "two parallel planes" for a cubic law conductance to be assigned for 2D case, and rectangular crosssectional conductance to be assigned for pseudo $3 \mathrm{D}$ case, where fluid flows along the $\mathrm{x}$ axis. (d) The illustration of how the linear set of equations to be defined for each pore element.

Forthe2D case, Figure6 illustrates how the poreelement is transformed intopseudo-3Drectangular-duct first and then modelled as "two parallel planes" to assign the conductance. The pore space of each pore element is partitioned by the radical axes with the neighbouring elements, which is a line perpendicular to the line connecting the centres of two non-concentric circles. Corresponding to the constant z-direction velocity used in 2D case, weassume each poreelement is "two infinite parallel planes", with aperture and length equal to $2 \cdot r_{m b}$. We adopt the cubic law equation for parallel planes to assign the conductance for each pore element:

$$
\mathrm{g}=\frac{\begin{array}{c}
\text { width } \cdot(2 \cdot \\
\left.\mathrm{r}_{m b}\right)^{3}
\end{array}}{12 \mu}
$$

The choice of width has no influence on the permeability calculation for $2 \mathrm{D}$ case because the width will be cancelled when Darcy's law is applied to find permeability. We use an arbitrary value of width $=1000 \mu \mathrm{m}$. 
For the $3 \mathrm{D}$ case, the hydraulic conductance of each pore element is generally correlated with its cross-sectional area and shape factor; the analytical expressions were derived by Patzek and Silin. ${ }^{37}$ Table 1 shows the conductance equations used for pore elements with different shape factors. The shape factor is defined as $\mathrm{G}=\mathrm{A} / \mathrm{p}^{2}$ and $\hat{\mathrm{G}}=16 \mathrm{G}$; the latter is for the simplification of the conductance equation of a rectangular shape. Each pore element has a length of $2 \cdot \mathrm{r}_{m b}$, while its cross-sectional area and shape factor depend on its geometry. We note that existing pore network models only use triangular-square-circle as the equivalent shapes of pores or throats. Hence when assigning the conductance, the shape factor of the pores or throats is first computed before using it in the appropriate conductance equation. In the current study, however, we use the conductance equation of a rectangular shape as explained in the section of "Validation of pore network results".

Table 1: The hydraulic conductance equations used to assign to the pore elements

\begin{tabular}{|c|c|c|}
\hline Equivalent shape & hydraulic conductance & shape factor range \\
\hline triangular & $\mathrm{g}=0.6 \cdot \frac{A^{2} G}{\mu}$ & $G \subseteq 0, \frac{3}{36}$ \\
\hline square & $\mathrm{g}=0.5623 \cdot \frac{A^{2} G}{\mu}$ & $\mathrm{G} \subseteq \frac{3}{36}, \frac{1}{16}$ \\
\hline circular & $\mathrm{g}=0.5 \cdot{ }_{\mu}^{A^{2} G}$ & $\mathrm{G} \subseteq{ }_{\left.\frac{1}{16}, \frac{1}{4 \pi}\right]}$ \\
\hline reetangular & $g=\frac{0.5623 A^{2}(}{16 \mu} 0.3214 \hat{G}^{3}+0.063 \hat{G}^{2}+0.6109 \hat{G}^{)}$ & $\hat{G} \subseteq(0,1]$ \\
\hline
\end{tabular}

\section{Results and discussions}

In this section, we demonstrate that thepore structures quantified by the new method can be used to provide a qualitatively reasonable estimate of the permeability of rock samples for a North Sea sandstone reservoir. Wefirstly analyse the geometrical and topological properties computed from SEM images of several rock samples using our quantification method. We also show the image resolution effect on the quantified pore structure properties. Then, we 
simulate the single phase flow in the pore elements network extracted from different but 
interconnected test images and compare the results with that of finite element method.

\section{Analysis of geometrical properties}

The rock samples studied in this paper were obtained from a North Sea sandstone reservoir which has a long diagenetic history and accordingly a complex pore structure. The online supplement materials contain the high resolution SEM images of the ten core plugs and a complete list of results including the core plug properties from laboratory. Table 2 shows the experimentally measured porosity and absolute permeability for the samples used in this paper. The sandstone samples were impregnated with resin under vacuum and then polished until optically flat. The polished samples were imaged by SEM at the University of Aberdeen Centre for Electron Microscopy, Analysis and Characterisation (ACEMAC). All recorded images were obtained at multi-scale from the backscattered electron (BSE) detector.

Table 2: Rock sample properties, the Helium porosity and measured air permeability

\begin{tabular}{lcc}
\hline \multicolumn{3}{l}{ Core plugs Identifier $\phi_{\text {lab }}(\%)$} \\
\hline 44 & 28.3 & 557 \\
93 & 17.6 & 100 \\
124 & 18.2 & 1.2 \\
130 & 24.5 & 23 \\
167 & 24.5 & 270 \\
367 & 16.2 & 44.5 \\
\hline
\end{tabular}

Three core plugs, 93, 130 and 124, are first selected to study the geometrical properties in terms of pore size distributions (PSD). The pore element quantification results and SEM images are shown in Figure 7. The corresponding pore size and cumulative PSD curves are shown in Figure 8, respectively. Plug 93 has the bigger pores visually from its rock image compared to the other two samples, and this trend can also be easily inferredfrom their cumulative PSD curves. PSD curves show a characteristically log-normal distribution, therefore the cumulative distribution function is a kind of complementary error function. The 

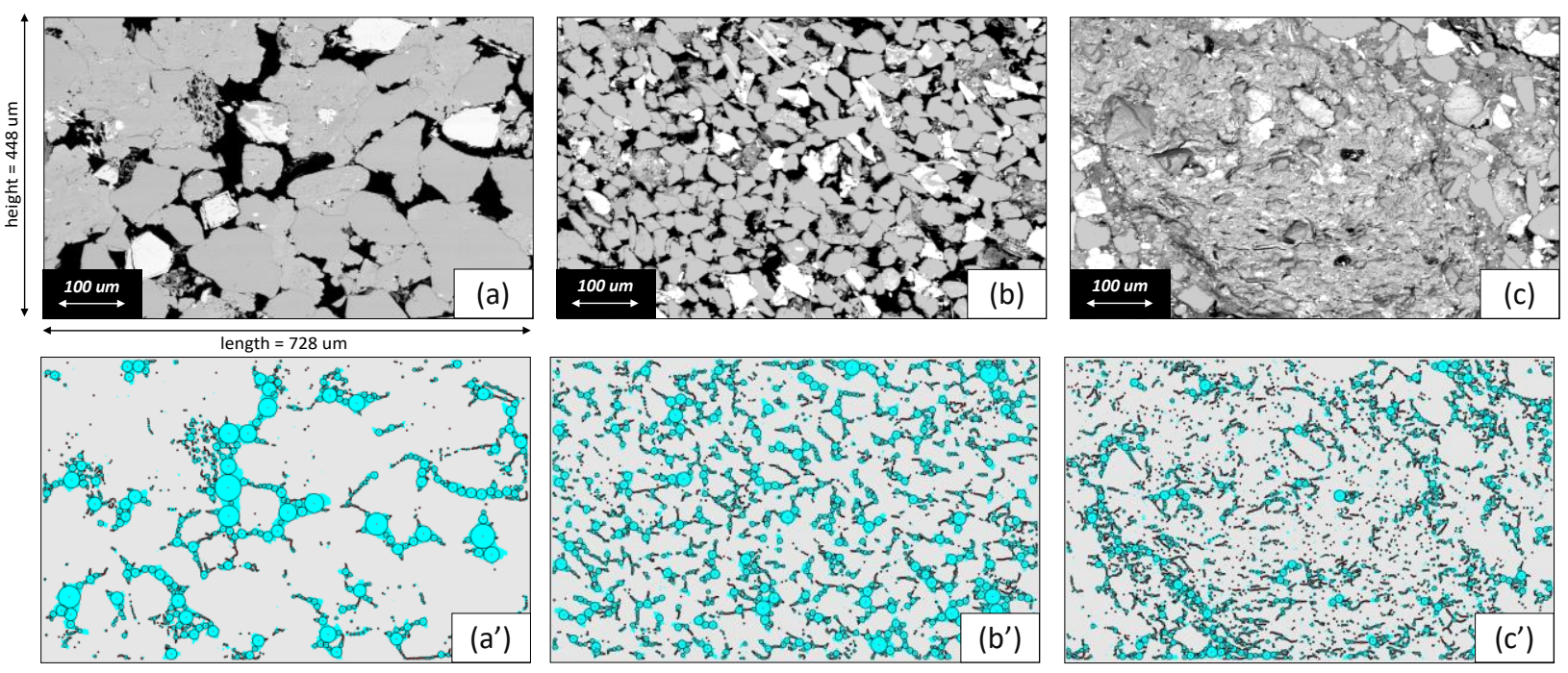

Figure 7: SEM images of three core plugs are analysed by the new method. The top are SEM images and bottom are the corresponding pore elements quantification results shown in binary images after preliminary processes. (a) and (a'). Plug 93, (b) and (b'). plug 130, (c) and (c') plug 124.

geometric mean pore radius and standard deviation for plug 93, 130 and 124 are given in Table 3. It shows that the larger pore size is, the higher the absolute permeability. However, bigger pore size does not guarantee higher permeability since permeability is also affected by pore structure connectivity.

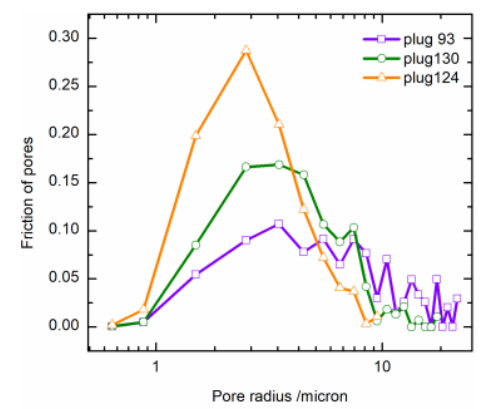

(a)

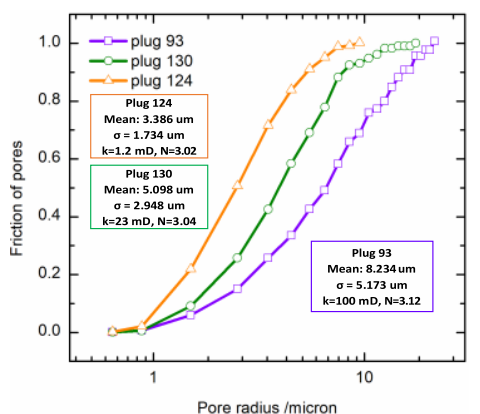

(b)

Figure 8: (a) Pore size distribution (PSD) curves quantified from SEM rock images of core samples 93,130 and 124. (b)Corresponding cumulative PSDcurves. Thegeometric mean radius, standard deviation $(\sigma)$ and average coordination number $(\mathrm{N})$ from the current method, absolute permeability (k) from laboratory measurement are reported.

In addition, Figure 9 shows the size distributions between the smallest pore elements on the medial axis chains and the local minima (pore throats). The relative differences 


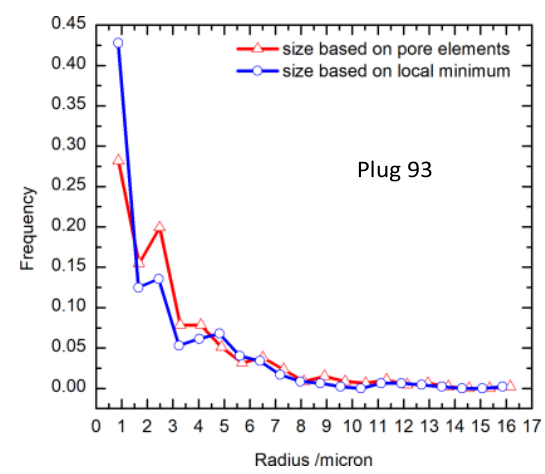

(a)

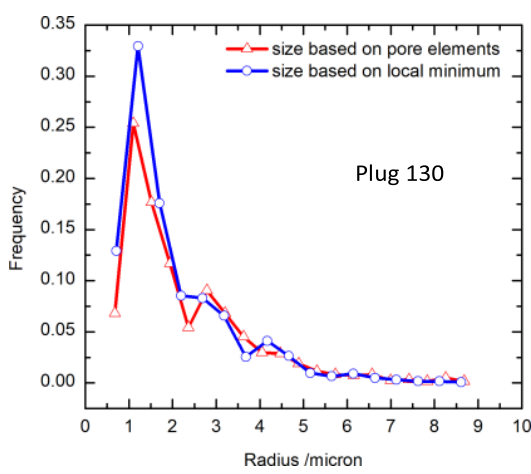

(b)

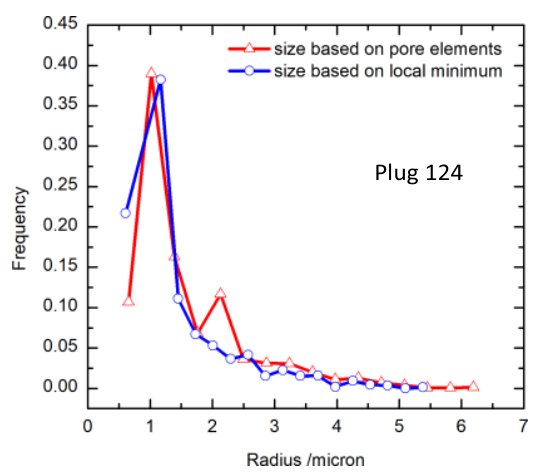

(c)

Figure 9: The comparisons of size distributions between the smallest pore elements on the medial axis chains and the local minima (pore throats) for core samples (a) plug 93, (b) plug 130, and (c) plug 124.

between them are low, showing that the current method provides a good approximation of pore throats.

Another threecoresamples, 44, 167 and 367, combining with plug 130, are selected to find the correlation of $\Phi(r)$ as a function of pore radius r. Figure 10 shows the complementary cumulative probability distribution curves for these samples. We chose such samples because their Helium porosity and measured air permeability cover wider range of properties.

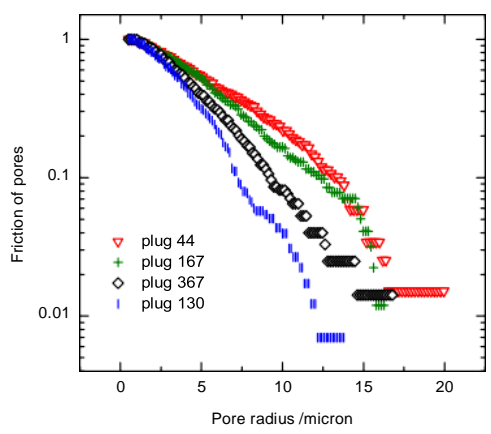

Figure 10: Cumulative PSD curves of core plugs 44, 167, 367 and 130 used to determine the cumulative probability $\Phi(r)$ as a function of pore radius $r$.

Weobserve a quadratic relationship between $\log (\Phi(\mathrm{r}))$ and $\mathrm{r}$ with an exponential cut-off at larger pore radii. The selection of the cut-off radius is based on the criterion that the correlation coefficient satisfies $\mathrm{R}^{2}>0.99$ in all cases when fitting the cumulative PSD curves. We have 
fitted the data to an empirical correlation and the parameters are given in Tables 3.

$$
\Phi(\mathrm{r})=\mathrm{a} \cdot \mathrm{e}^{-b\left(\frac{r}{0}+c\right)^{2}}
$$

Table 3: The geometric mean radius, standard deviation and average coordination number from core sample SEM images and the fitted parameters for cumulative pore size distribution

\begin{tabular}{lcccccccc}
\hline Core plugs & $\mathrm{r}_{\text {mean }}(\mu \mathrm{m})$ & $\sigma(\mu \mathrm{m})$ & $\mathrm{N}$ & $\mathrm{R}^{2}$ & $\mathrm{r}_{0}(\mu \mathrm{m})$ & $\mathrm{a}$ & $\mathrm{b}$ & $\mathrm{c}$ \\
\hline 44 & 8.246 & 5.150 & 3.38 & 0.9982 & 12.21 & 2.55 & 0.61 & 1.18 \\
93 & 8.234 & 5.173 & 3.11 & 0.9974 & 13.60 & 8.09 & 0.38 & 2.29 \\
124 & 1.734 & 1.734 & 3.12 & 0.9952 & 4.62 & 9.35 & 0.50 & 1.96 \\
130 & 5.098 & 2.948 & 3.08 & 0.9962 & 6.88 & 1.22 & 1.32 & 0.29 \\
167 & 6.878 & 4.064 & 3.26 & 0.9976 & 10.80 & 2.11 & 0.81 & 0.87 \\
367 & 5.722 & 3.354 & 3.30 & 0.9986 & 8.65 & 1.80 & 0.92 & 0.70 \\
\hline
\end{tabular}

In addition, we calculate the characteristic pore element radius from the cumulative PSD curves to predict the absolute permeability based on an empirical correlation: ${ }^{38}$

$$
\mathrm{k}_{\text {calc }}=\frac{\left(2 \cdot \mathrm{r}_{c}\right)^{2}}{226 \cdot \mathrm{F}}
$$

Here, we obtain the characteristic pore element radius from the cumulative PSD curves where the cumulative probability is $80 \%$, i.e., only $20 \%$ of pore elements are larger than this radius. The cumulative probability and corresponding characteristic radius are given in Table 4. We use the laboratory measured special core analysis (SCAL) formation factors from $3 \mathrm{D}$ samples.

Table 4: The parameters used to predict the permeability from rock sample images

\begin{tabular}{lccccc}
\hline \multicolumn{5}{l}{ Core plugs $\Phi(\mathrm{r})(\%) \mathrm{r}_{c}(\mu \mathrm{m}) \mathrm{F} \mathrm{k}_{\text {calc }}(\mathrm{mD}) \mathrm{k}_{\text {meas }}(\mathrm{mD})$} \\
\hline 44 & 79.35 & 10.43 & 8.3 & 232.13 & 557 \\
93 & 79.49 & 11.84 & 19.17 & 129.48 & 100 \\
124 & 79.07 & 3.67 & 18.18 & 13.12 & 1.2 \\
130 & 79.52 & 6.21 & 10.92 & 62.41 & 23 \\
167 & 80.11 & 8.88 & 10.23 & 136.28 & 270 \\
367 & 79.92 & 7.32 & 21.46 & 44.19 & 44.5 \\
\hline
\end{tabular}


The only parameter used from 2D images is the characteristic pore size. As the research of Rabbani et al. ${ }^{39}$ shows, the pore sizes between $2 \mathrm{D}$ and $3 \mathrm{D}$ rock samples are similar. Hence the predicted permeability can be compared with the measured air permeability.

Figure 11 shows a plot of the calculated permeability against the measured air permeability. With the exception of core plug 124, there is in general a reasonable agreement between the predicted and measured permeability. The major discrepancy for core plug 124 is likely due to the heterogeneity within the core which is not captured by the SEM imaging.

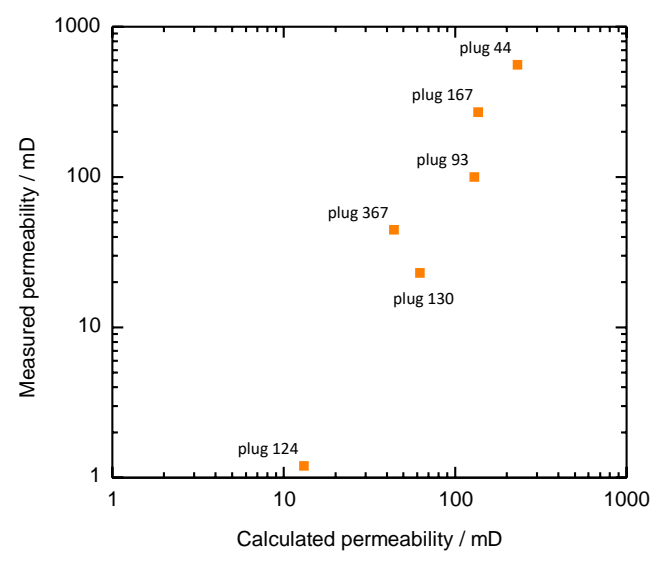

Figure 11: Calculated permeability from core sample SEM images against measured air permeability

This analysis shows that the characteristic pore radius calculated from this method, combined with a simple empirical correlation, can provide an approximate estimate of the permeability. However, we expect that a 3D pore network simulation will give a more reliable estimate of permeability.

\section{Analysis of topological properties}

Topological properties of pore structure are of vital importance to the transport properties because they affect the connectivity of the fluid flow. Average coordination number $(\mathrm{N})$ is used to quantify pore structure connectivity, ${ }^{1}$ which represents the average number of bonds connected with nodes, namely, $\mathrm{N}=2 \cdot \mathrm{N}_{b} / \mathrm{N}_{n}$. The plot of average coordination number and permeability is shown in Figure 12(a). Generally speaking, it is expected that higher 
coordination number has better connectivity and result in higher permeability. This can be seen from the trend in Figure 12(a) where the coordination number and permeability (red triangular dots) have a good positive relationship. In addition, as shown in Figure 12(b), cumulative PSD curves for plug 44 and 93 are close and almost overlapped, and geometric pore radius for plug 44 and 93 are $8.246 \mu \mathrm{m}$ and $8.234 \mu \mathrm{m}$ respectively, but coordination number of plug $44(\mathrm{~N}=3.38)$ is larger than plug $93(\mathrm{~N}=3.11)$, thus the permeability of plug 44 is higher than for plug 93.

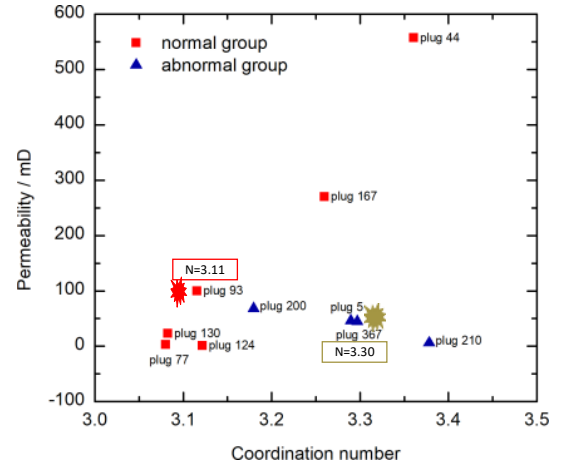

(a)

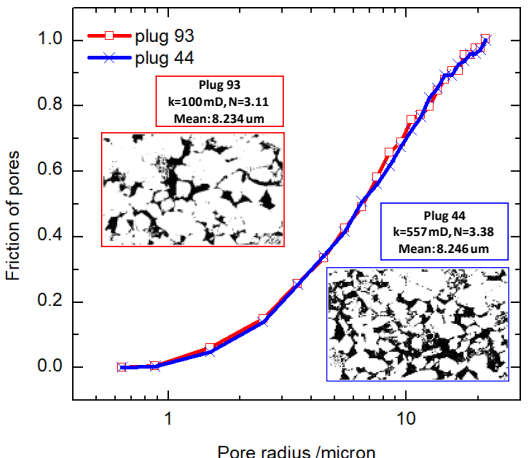

(b)

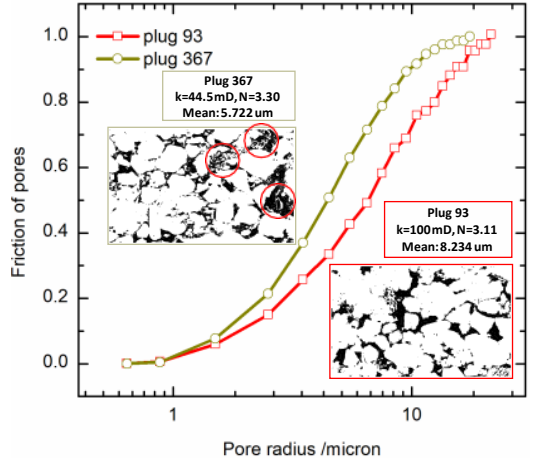

(c)

Figure 12: (a) Relationship between the coordination number $(\mathrm{N})$ and the measured air absolute permeability (k), plug 93 and 367 are chosen as an example to explain the reasons of abnormal trend of $\mathrm{N}-\mathrm{k}$ plot. (b) and (c) Cumulative PSD curves and corresponding SEM images for plugs 44 and 93 (b) and plugs 93 and 367 (c), where the red ellipses emphasize the grains filling pores.

However, larger coordination number does not always guarantee higher permeability, for instance, the sample 367 and 93. Figure 12(c) shows that the pore size of plug 93 is larger than plug 367 as well as its permeability. However, the coordination number of plug 93 $(\mathrm{N}=3.11)$ is lower than plug $367(\mathrm{~N}=3.30)$. The reason is plug 367 has many small grains filling the larger pores, which are likely caused by clay filling or secondary diagenesis. This yields the abnormally high coordination number of plug 367 but reduces the pore size and lowers the permeability. This result shows the higher coordination number alone does not guarantee higher permeability as it might be due to micro-pores.

Figure 13 shows the quantitative analysis on the connectivity over pore sizes of plug 93 and 367 . We observe that the frequency distribution of coordination number of plug 
93 is shifted to the left compared to plug 367, indicating plug 93 has smaller coordination number consistent with the average coordination number. The mean and standard deviation of coordination number versus pore sizes are plotted in Figure 13 (b) and (c). The mean and standard deviation of the coordination number increase with pore radius. We also observe a linear trend for both samples when the pore size is less than $15 \mu \mathrm{m}$ in the log-log plot.

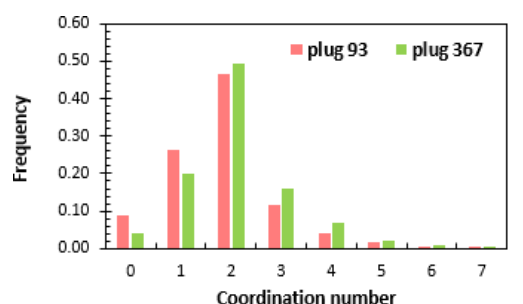

(a)

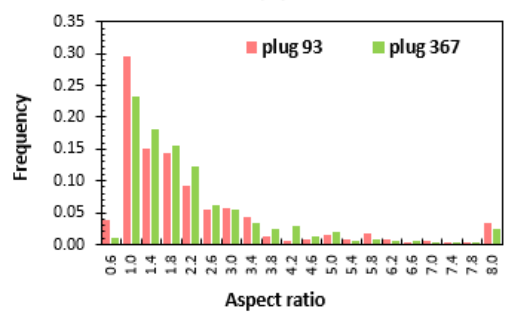

(d)

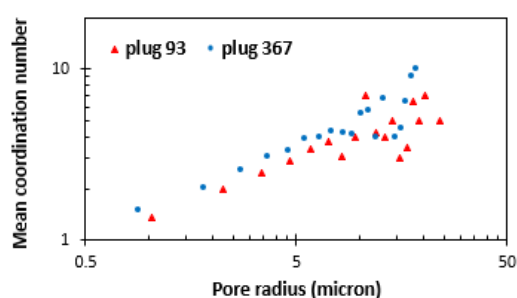

(b)

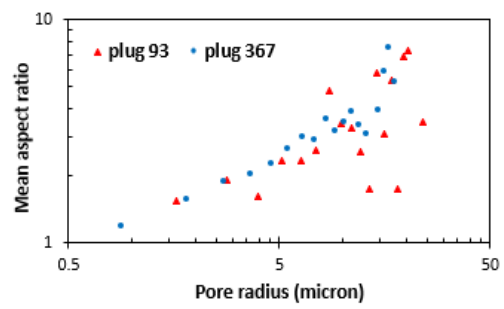

(e)

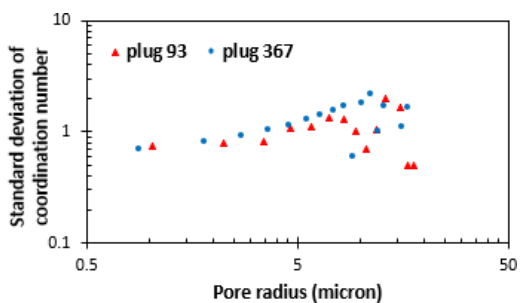

(c)

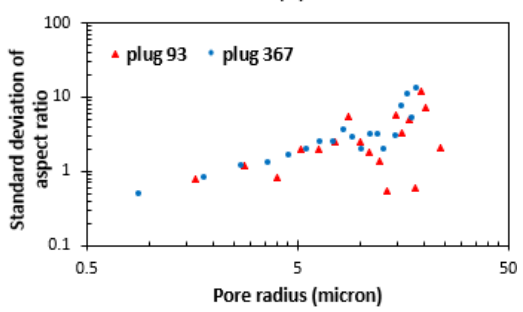

(f)

Figure 13: The connectivity analysis of the pore structures for plug 93 and 367. (a) Coordination number frequency distribution. (b) Mean coordination number vs. pore radius. (c) Standard deviation of coordination number vs. pore radius. (d) Aspect ratio frequency distribution. (e) Mean aspect ratio vs. pore radius. (f) Standard deviation of aspect ratio vs. pore radius

In addition, the connectivity of larger junction pores and smaller throat pores is important to the fluid flow, which can be quantifies by aspect ratio, i.e., the ratio of pore radius to throat radius. Figure 13 (d) to (f) give the results that are related to aspect ratio and pore sizes. Plug 367 has a bit higher aspect ratio than plug 93 since it has more smaller throats radii except for some larger pores. Both samples show that the mean and standard deviation of the aspect ratio increase with the pore sizes, as shown in Figure 13 (e) and (f). However, there is no much difference between the mean aspect ratio and pore sizes for these two samples. 


\section{Effects of image resolution}

Figure 14 shows the three different resolutions of the SEM images for plug 5, which are used to explore the resolution effect on quantified pore structure properties, the results are given in Table 5 . The high resolution image has a better spatial resolution and the identified minimum pore size is smaller and therefore we see the pore size distributions shifted to the left. The number of smaller pores $(r<1.0 \mu \mathrm{m})$ recognised by the higher resolution image is approximate $8.7 \%$ more than that of lower resolution image. The largest pore identified by different resolution images does not change too much. However, the average coordination number of high resolution image $(\mathrm{N}=2.97)$ is higher than that of the low resolution image $(\mathrm{N}=1.85)$. This is because smaller resolved grains filling in the larger pores can be recognized by high resolution images and this introduces more medial axis branches. This conclusion is different from Liang et al. ${ }^{35}$ whose result shows that resolution of images does not affect the topology of medial axis.

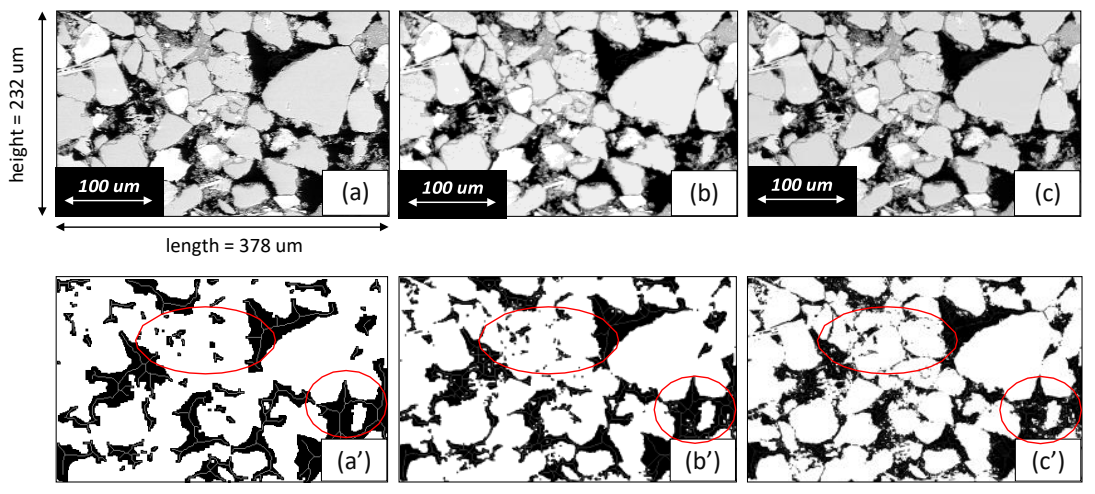

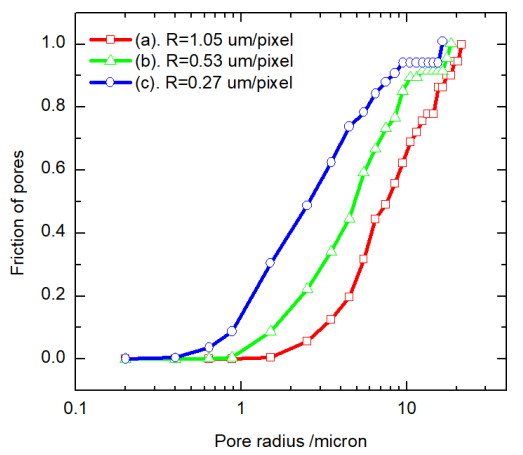

(d)

Figure 14: SEM images (top) for plug 5 at same position but with different resolutions and the corresponding binary images with medial axis after preliminary processes (bottom), (a) and (a') resolution is $1.05 \mu \mathrm{m} /$ pixel, (b) and (b') resolution is $0.52 \mu \mathrm{m} /$ pixel, (c) and (c') resolution is $0.27 \mu \mathrm{m} /$ pixel; and (d) the cumulative PSD curves.

\section{Validation of pore network results}

Figure 15 shows three test images used to validate our pore element network model. Image A is a SEM image of a real micro model that was used to study the transport of colloids in 
Table 5: Results of the different resolution images for plug 5

\begin{tabular}{ccccc}
\hline $\mathrm{R}(\mu \mathrm{m} /$ pixel $)$ & $\mathrm{r}_{\text {mean }}(\mu \mathrm{m})$ & $\mathrm{r}_{\max }(\mu \mathrm{m})$ & $\mathrm{r}_{\text {min }}(\mu \mathrm{m})$ & $\mathrm{N}$ \\
\hline 1.05 & 9.5 & 17.9 & 1.05 & 1.85 \\
0.53 & 6.5 & 17.9 & 0.53 & 2.67 \\
0.27 & 4.4 & 16.3 & 0.27 & 2.97 \\
\hline
\end{tabular}

porous medium, ${ }^{40}$ while $\mathrm{B}$ and $\mathrm{C}$ are synthetic images. Using the methodology described above, these images were used to construct the pore element networks to simulate single phase flow and compute absolute permeability, see Figure 15(b). In addition, the same image was meshed by finite elements to solvethe Stokes equation and mass balance equation using the COMSOL Multiphysics module (v5.3). ${ }^{41}$ Then the inlet flux was computed, and an application of Darcy's Law yields the permeability.

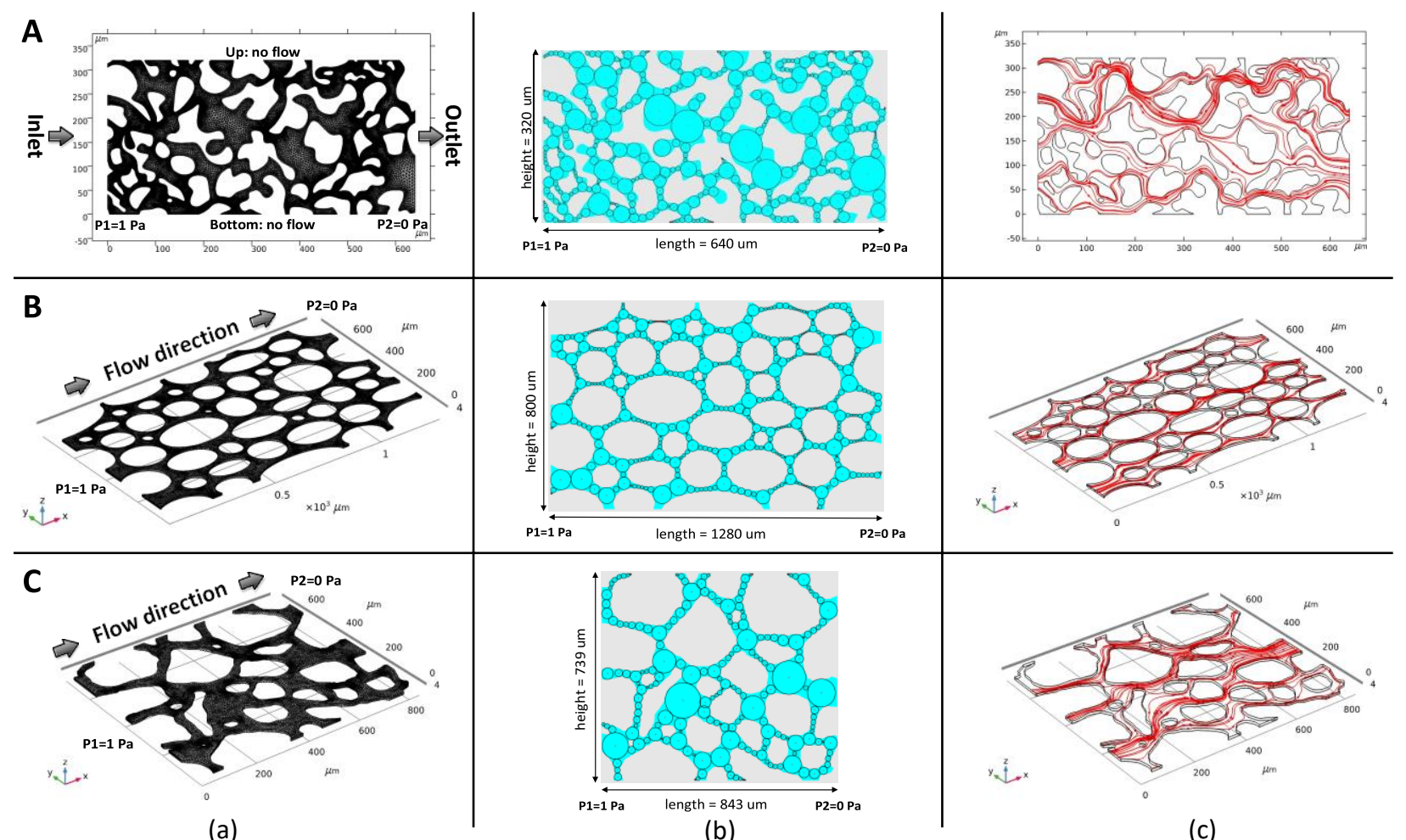

(a)

(b)

(c)

Figure 15: The validation of pore element networks by finite element method for three images; the image resolution of A is $0.39 \mu \mathrm{m} /$ pixel, and $1.0 \mu \mathrm{m} /$ pixel for B and C. (a) The mesh for the three images; image A is meshed with COMSOL, while images B and C are meshed with ScanIP. (b) The corresponding pore element networks quantified by the method in this paper. (c) Velocity streamline simulated by COMSOL. 
Table 6: Results calculated from finite element method and pore element network model respectively

\begin{tabular}{lcccccc}
\hline \multirow{2}{*}{ Value } & \multicolumn{2}{c}{ Image A } & \multicolumn{2}{c}{ Image B } & \multicolumn{2}{c}{ Image C } \\
\cline { 2 - 7 } & FEM & PNM & FEM & PNM & FEM & PNM \\
\hline Permeability (mD) & 4542.80 & 4774.43 & 682.74 & 711.74 & 955.61 & 948.62 \\
Deviation (\%) & - & 5.10 & - & 4.25 & - & -0.73 \\
Number of elements & 50,152 & 361 & 109,910 & 998 & 111,228 & 421 \\
CPU time (seconds) & 62 & 0.16 & 5935 & 0.73 & 7355 & 0.25 \\
\hline
\end{tabular}

The mesh of image A is a 2D mesh and downloaded from COMSOL website. ${ }^{41}$ However, we could not obtain the effective mesh for image B and C by using COMSOL alone, hence Synopsys Simpleware ${ }^{\mathrm{TM}}$ ScanIP and FE module were used for CFD mesh generation. Because ScanIP can only deal with $3 \mathrm{D}$ images, a stack of 10 same images was created for image $\mathrm{B}$ and $\mathrm{C}$ and imported into scanIP to generate the mesh.

There is a difference when we assign the hydraulic conductance for the pore elements in images A, B and C. Image A is used as a 2D case, hence we assume each pore element is a pseudo-3D-rectangular-duct with a very larger width (z coordinate) and we adopt the cubic law shown in equation 7 to assign the conductance to each pore element. However, images $\mathrm{B}$ and $\mathrm{C}$ are treated as $3 \mathrm{D}$ cases and the cross-sectional area of each pore element is rectangular, hence we adopt the conductance equation of a rectangular cross-sectional shape for the pore elements in images B and C, as shown in Table 1. The elements have height and length of $2 \cdot r_{m b}$ and the same width as the $3 \mathrm{D}$ images, here width $=10 \mu \mathrm{m}$.

Table 6 presents the permeability estimates for the three test images. The absolute permeabilities calculated by pore element network method show excellent agreement with finite element method. In addition, on a desktop PC (Intel Core i5 CPU, 8GB RAM), the computation time for the pore network model is much faster than that for the corresponding finite element solution, see table 6 . 


\section{Conclusions}

We have presented a new pore structure quantification method, combining with pore network extraction work-flow which discretizes the pore space into a set of pore elements. This method combines the advantages of the maximal ball and medial axis methods but overcomes their problems. By employing the maximal ball concept, pore bodies are defined sufficient to coincide with the geometrical pores. The method also uses the medial axis transformation to preserve the topology of the pore structure. The method naturally divides the pore space into connected pore elements with basic geometrical properties, which can be used to construct pore networks.

This work shows that the important factors controlling the fluid flow are the large/small features of pore structure as well as their connectivity. It is less consequential to explore the definition or boundary between the pore bodies and throats. We suggest that the distinction between pore bodies and throats could be discarded in favour of connected pore elements for the simulation of fluid flow. Hence this new method employs the equivalent pore elements to capture the features of pore structure from the widest to the narrowest part in the pore space, honouring the topology without over-segmenting the pore space. The main advantages of this new method mainly represents these following features: it is a higher level of discretization of the pore space, it can capture large/small features of pore structure and it can predict the permeability accurately compared to the other traditional methods, which construct throats explicitly.

We have demonstrated the utility of the method by applying it to a set of SEM images of core samples from a heterogeneous sandstone reservoir. The cumulative pore size distribution and coordination number can be employed to quantitatively analyse the influence of pore structure properties on absolute permeability. The larger pore size results in higher absolute permeability, and vice versa. For similar pore size distribution, larger coordination number gives rise to higher permeability. A special case occurs with larger coordination number but smaller pore size, which yield lower permeability possibly due to the existence of micro- 
porosity.

The resolution effect of SEM images on quantified pore structures properties were analysed. Higher resolution images recognise more smaller pores and larger coordination number, however, the largest pore recognised by different resolution images does not change too much. We also validated the method by comparing extracted pore network single phase flow simulation results with that of finite element method.

For future work, we plan to extend this method to $3 \mathrm{D}$ images. We will compare this method with the existing pore network extraction techniques, such as the maximal ball method, medial axis method and watershed segmentation method, ${ }^{8,10,23,39}$ in terms of the distribution of geometrical and topological properties as well as the results of single and two phase simulation. Wewill also investigate the correlation of geometrical and topological properties between $2 \mathrm{D}$ and $3 \mathrm{D}$ images.

\section{Acknowledgement}

CW thanks China Scholarship Council's financial support for his Ph.D. study. The authors thank Anasuria Operating Company Limited (AOC) for making available the core plug samples. We thank the two reviewers for their insight comments and constructive suggestions towards improving our manuscript. Finally, we thank John Still of University of Aberdeen Centre for Electron Microscopy, Analysis and Characterisation (ACEMAC) for invaluable assistance in the acquisition of SEM images.

\section{Supporting Information Available}

The following files are available and free of charge.

- Images: high resolution SEM images of the ten rock samples and three test images

- Rock sample properties.xlsx: rock sample properties measured from laboratory and 
method described in this paper

\section{References}

(1) Blunt, M. J.; Bijeljic, B.; Dong, H.; Gharbi, O.; Iglauer, S.; Mostaghimi, P.; Paluszny, A.; Pentland, C. Pore-scale imaging and modelling. Adv. Water Resour. 2o13, 51, 197216.

(2) Blunt, M. J. Multiphase Flow in Permeable Media: A Pore-Scale Perspective; Cam- bridge University Press: Cambridge, 2017.

(3) Chen, S.; Doolen, G. D. Lattice Boltzmann Method. Annu. Rev. Fluid Mech. 1998,30, 329-364.

(4) Prodanovic, M.; Bryant, S. L. A level set method for determining critical curvatures for drainage and imbibition. J. Colloid Interface Sci. 2oo6, 304, 442-458.

(5) Raeini, A. Q. Modelling multiphase flow through micro-CT images of the pore space. Ph.D. thesis, Imperial College London, 2013.

(6) Okabe, H.; Blunt, M. J. Pore space reconstruction of vuggy carbonates using microtomography and multiple-point statistics. Water Resour. Res. 2007, 43, 3-7.

(7) Wu, K.; Van Dijke, M. I. J.; Couples, G. D.; Jiang, Z.; Ma, J.; Sorbie, K. S.; Craw- ford, J.; Young, I.; Zhang, X. 3D Stochastic Modelling of Heterogeneous Porous Media Applications to Reservoir Rocks. Transp. Porous Media 2006, 65, 443-467.

(8) Jiang, Z.; Wu, K.; Couples, G.; Van Dijke, M.; Sorbie, K. S.; Ma, J. Efficient extraction of networks from three-dimensional porous media. Water Resour. Res. 2007, 43, 1-17.

(9) Wu, K.; Jiang, Z.; Couples, G. D.; Van Dijke, M.; Sorbie, K. S. Reconstruction of Multi-Scale Heterogeneous Porous Media and Their Flow Prediction. Int. Symp. Soc. Core Anal. 2007, 1-12. 
(10) Dong, H.; Blunt, M. J. Pore-network extraction from micro-computerized-tomography images. Phys. Rev. E 2009, 80, 1-11.

(11) Zhou, Y.; Hatzignatiou, D. G.; Helland, J. O. On the estimation of $\mathrm{CO} 2$ capillary entry pressure: Implications on geological $\mathrm{CO} 2$ storage. Int. J. Greenh. Gas Control 2017, 63, 26-36.

(12) Li, Y.; Wu, P.; Liu, W.; Sun, X.; Cui, Z.; Song, Y. A microfocus X-ray computed tomography based gas hydrate triaxial testing apparatus. Rev. Sci. Instrum. 2019, 90, 055106.

(13) Valvatne, P. H.; Blunt, M. J. Predictive pore-scale modeling of two-phase flow in mixed wet media. Water Resour. Res. 2004, 40, 1-21.

(14) Valvatne, P. H.; Piri, M.; Lopez, X.; Blunt, M. J. Predictive Pore-Scale Modeling of Single and Multiphase Flow. Transp. Porous Media 2005, 58, 23-41.

(15) Zhou, Y.; Helland, J. O.; Hatzignatiou, D. G. Computation of Three-Phase Capillary PressureCurvesand Fluid Configurations at Mixed-WetConditionsin 2D Rock Images. SPE J. 2016, 21, 152-169.

(16) Prodanovic, M.; Bryant, S. L. Investigating Matrix/Fracture Transfer via a Level Set Method for Drainage and Imbibition. SPE J. 2010, 125.

(17) Cai, J.; Yu, B. A Discussion of the Effect of Tortuosity on the Capillary Imbibition in Porous Media. Transp. Porous Media 2011, 89, 251-263.

(18) Lindquist, W. B.; Lee, S.-M.; Coker, D. A.; Jones, K. W.; Spanne, P. Medial axis analysis of void structure in three-dimensional tomographicimages of porous media.J. Geophys. Res. Solid Earth 1996, 101, 8297-8310. 
(19) Raeini, A. Q.; Bijeljic, B.; Blunt, M. J. Generalized network modeling: Network extraction as a coarse-scale discretization of the void space of porous media. Phys. Rev. E 2017, 96, 013312.

(20) Scott, G.; Wu, K.; Zhou, Y. Multi-scale Image-Based Pore Space Characterisation and Pore Network Generation: Case Study of a North Sea Sandstone Reservoir. Transp. Porous Media 2019, 1-30.

(21) Thompson, K. E.; Willson, C. S.; White, C. D.; Nyman, S.; Bhattacharya, J.; Reed, A. H. Application of a New Grain-Based Reconstruction Algorithm to Microtomography Images for Quantitative Characterization and Flow Modeling. SPE J. 2005, 12.

(22) Rabbani, A.; Jamshidi, S.; Salehi, S. An automated simple algorithm for realistic pore network extraction from micro-tomography images. J.Pet. Sci. Eng. 2014, 123, 164171.

(23) Gostick, J. T. Versatile and efficient pore network extraction method using markerbased watershed segmentation. Phys. Rev. E 2017, 96, 023307.

(24) Silin, D.; Patzek, T. Pore space morphology analysis using maximal inscribed spheres. Phys. A Stat. Mech. its Appl. 2oo6, 371, 336-36o.

(25) Bultreys, T.; De Boever, W.; Cnudde, V. Imaging and image-based fluid transport modeling at the pore scale in geological materials: A practical introduction to the current state-of-the-art. Earth-Science Rev. 2016, 155, 93-128.

(26) Thovert, J.F.; Salles, J.; Adler, P.M. Computerized characterization of the geometry of real porous media: their discretization, analysis and interpretation. J. Microsc. 1993, 170, 65-79. 
(27) Al-Kharusi, A. S.; Blunt, M. J. Network extraction from sandstone and carbonate pore space images. J. Pet. Sci. Enginering 2007, 56, 219-231.

(28) Sheppard, A. P.; Sok, R. M.; Averdunk, H.; Robins, V. B.; Ghous, A. Analysis of Rock Microstructure Using High Resolution X-ray Tomography. Proc. Int. Symp. Soc. Core Anal. Trondheim, Norway, 2006.

(29) Wildenschild, D.; Sheppard, A. P.X-ray imaging and analysis techniques for quantifying pore-scale structure and processes in subsurface porous medium systems. Adv. Water Resour. 2013, 51, 217-246.

(30) Chen, Y.; Hsu, W. A modified fast parallel algorithm for thinning digital patterns. Pattern Recognit. Lett. 1988, 7, 99-106.

(31) She, F. H.; Chen, R. H.; Gao, W. M.; Hodgson, P. D.; Kong, L. X.; Hong, H. Y. Improved 3D thinning algorithms for skeleton extraction. 2009 Digit. Image Comput. Tech. Appl. 2009; pp 14-18.

(32) Iassonov, P.; Gebrenegus, T.; Tuller, M. Segmentation of X-ray computed tomography images of porous materials: A crucial step for characterization and quantitative analysis of pore structures. Water Resour. Res. 2009, 45 .

(33) Schlüter,S.; Sheppard, A.; Brown, K.; Wildenschild, D. Imageprocessing of multiphase images obtained via X-ray microtomography: A review. Water Resour. Res. 2014, 50, $3615-3639$.

(34) Bradski, G. The OpenCV Library. Dr. Dobb's J. Softw. Tools 2ooo,

(35) Liang, Z.; Ioannidis, M.; Chatzis, I. Geometric and Topological Analysis of ThreeDimensional Porous Media: Pore Space Partitioning Based on Morphological Skeletonization. J. Colloid Interface Sci. 2000, 221, 13-24. 
(36) Øren, P.-E.; Bakke, S. Process based reconstruction of sandstones and prediction of transport properties. Transp. Porous Media 2oo2, 46, 311-343.

(37) Patzek, T.; Dmitriy B. Silin, Shape Factor and Hydraulic Conductance in Noncircular Capillaries I. One-Phase Creeping Flow. J. Colloid Interface Sci. 2001, 304, 295-304.

(38) Katz, A. J.; Thompson, A. H. Quantitative prediction of permeability in porous rock. Phys. Rev. B 1986, 34, 8179-8181.

(39) Rabbani, A.; Ayatollahi, S.; Kharrat, R.; Dashti, N. Estimation of 3-D pore network coordination number of rocks from watershed segmentation of a single 2-D image. Adv. Water Resour. 2016, 94, 264-277.

(40) Sirivithayapakorn, S.; Keller, A. Transport of colloids in saturated porous media : A pore-scale observation of the size exclusion effect and colloid acceleration. Water Resour. Res. 2003, 39 .

(41) COMSOL AB, COMSOL Multiphysics. 2018; www.comsol.com. 